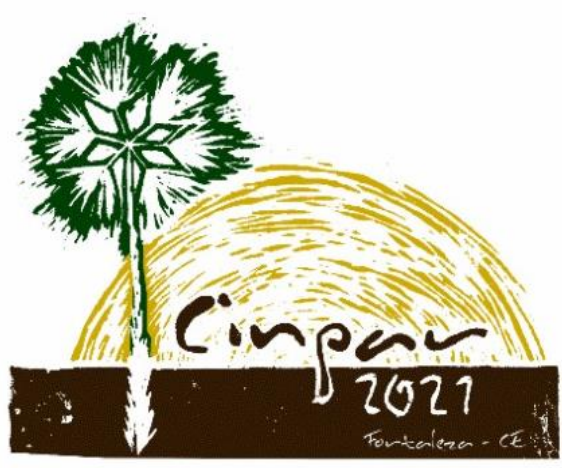

XVII Congresso Internacional sobre Patologia e Reabilitação das Construções

XVII Congreso Internacional sobre Patología y Rehabilitación de las Construcciones

XVII International Conference on Pathology and Constructions Rehabilitation

FORTALEZA (Brasil), 3 a 5 de junho de 2021

https://doi.org/10.4322/CINPAR.2021.119

\title{
Aplicação sustentável da fibra do babaçu como isolamento acústico em painéis de gesso
}

\author{
Osilene DOS SANTOS ROCHA ${ }^{1}$, Adeildo CABRAL DA SILVA ${ }^{2}$, Geny GIL SÁ ${ }^{3}$
}

${ }^{1}$ Instituto Federal de Educação, Ciência e Tecnologia, Fortaleza, Brasil, osilene.dsrocha@gmail.com ${ }^{1}$

${ }^{2}$ Instituto Federal de Educação, Ciência e Tecnologia, Fortaleza, Brasil, cabral@ifce.edu.br ${ }^{2}$

${ }^{3}$ Instituto Federal de Educação, Ciência e Tecnologia, Fortaleza, Brasil, genygilsa60@ifce.edu.br

Resumo: Os ensaios da combinação da fibra do babaçu como agregado, ocupando espaço e reduzindo o volume de gesso devem contribuir à redução sonora com o meio externo. Para Rezende, Filho, Nascimento (2014) a isolação sonora se refere à redução da transmissão sonora entre ambientes através de materiais ou sistemas construtivos que funcionem como barreiras físicas à propagação das ondas. Este estudo objetivou avaliar a aplicação sustentável das fibras do babaçu a partir das propriedades de isolamento acústico com o gesso. 0 estudo foi realizado com a produção de painéis de gesso agregando as fibras do babaçu, com os ensaios específicos e adaptações das normas da ABNT NBR 12129:2017 e ABNT NBR 12127:1991, caracterização física do pó e da fibra do babaçu, ensaios mecânicos e as propriedades acústicas dos painéis segundo a norma da ABNT NBR 15 575. Com os resultados dos ensaios da inserção da fibra, coletou-se na trituração dois tipos de amostras de fibras maior(A) e menor(B) e, após estufa obteve-se na amostra B com 106,0, o que pode contribuir para menor teor de umidade durante agregação do gesso. Revelou-se resultados significativos da fibra e, o ensaio da granulometria atendeu o esperado, destacando na dimensão máxima, a amostra $B 4,74$ foi obtido a 0,75 , pois foi o primeiro valor maior que $5 \%$, logo representado na peneira de $4,75 \%$. Durante a produção, o tempo de cura dos painéis de gesso comprometeram a finalização da proposta dos ensaios acústicos. Dessa forma, o estudo propõe uma continuidade dos ensaios específicos a fim de atender facilmente os critérios estabelecidos na norma pertinente a ABNT NBR 12129:2017 e a norma ABNT NBR 12127:1991. Portanto, visando adequar as expectativas de ensaio acústico foi relevante a placa de gesso como alternativa sustentável de aproveitamento dos resíduos da fibra do babaçu.

Palavras-chave: gesso, babaçu, sustentabilidade, ensaio acústico.

Abstract: The tests of the combination of babassu fiber as aggregate, occupying space and reducing the volume of plaster must contribute to the sound reduction with the external environment. For Rezende, Filho, Nascimento (2014) sound insulation refers to the reduction of sound transmission between environments through materials or construction systems that function as physical barriers to the propagation of waves. This study aimed to evaluate the sustainable application of babassu fibers from the properties of acoustic insulation with plaster. The study was carried out with the production of plaster panels aggregating babassu fibers, with specific tests and adaptations of the standards of ABNT NBR 12129: 2017 and ABNT NBR 12127: 1991, physical characterization of babassu powder and fiber, mechanical tests and the acoustic properties of the panels according to ABNT NBR 15 575. With the results of the tests of the fiber insertion, two types of fiber samples were collected in the grinding (A) and smaller (B) and, after stove, it was obtained in the sample B with 106.0, which can contribute to lower moisture content during plaster aggregation. Significant results of the fiber were revealed and, the granulometry test met the expected, highlighting in the maximum dimension, the sample B 4.74 was obtained at 0.75 , since it was the first value greater than $5 \%$, soon represented in the sieve of $4.75 \%$. During production, the curing time of the plaster 
panels compromised the finalization of the acoustic testing proposal. Thus, the study proposes a continuity of specific tests in order to easily meet the criteria established in the relevant standard to ABNT NBR 12129: 2017 and the ABNT NBR 12127: 1991 standard. Therefore, in order to adjust the expectations of acoustic testing, plasterboard was relevant as a sustainable alternative to use babassu fiber residues.

Keywords: plaster, babassu, sustainability, acoustic test

\section{Introdução}

Com os demasiados avanços tecnológicos e ambientais, surge o desafio do ser humano se reinventar os modelos tradicionais. $\mathrm{O}$ uso de materiais combinados é uma prática antiga, que se protagonizou a medida que a imaginação humana foi se desenvolvendo ao longo dos tempos. A fibra do babaçu é um material de propriedades singulares, assim como o gesso, que quando combinado com outro material, pode agregar-se a este conferindo uma melhor qualidade ao produto final.

De acordo com o Oliveira (2013), o babaçu é o nome genérico de uma palmeira não cultivada e originária do Brasil classificada genericamente como Orbinya oleífera por botânicos, conhecida também como Orbinya speciosa, Orbinya mariana ou Orbinya phalerata. $\mathrm{O}$ autor ressalta que o mesocarpo ou poupa, massa que fica entre o epicarpo e endocarpo, representa $20 \%$ do peso do fruto, é rico em amido e fibras, é utilizado para fazer farinha, rações e biocombustíveis. Enquanto o epicarpo constitui $13 \%$ do peso do fruto, usado na biomassa para produção de biocombustível ou em queima direta em caldeiras. Em termos de valor da produção, até 2011, o babaçu era considerado o segundo produto florestal não madeireiro no país, após o açaí, alcançando naquele ano R\$ 142 milhões (IBGE, 2016).

As três frações componentes da casca (epicarpo, mesocarpo e endocarpo) têm alternativas diversas de uso, para as quais já existem tecnologias definidas, outras em fase de pesquisa e algumas por pesquisar, principalmente, da fase de escala piloto atá a industrial (EMBRAPA, 1984). Com base nas características do epicarpo e mesocarpo, buscou-se para o presente estudo o aproveitamento sustentável das fibras do babaçu como agregado na produção de placa de gesso a fim de testar melhor qualidade acústica do material.

Considerando a produção de gesso natural, segundo Rezende, Filho e Nascimento (2014) acontece basicamente em quatro etapas: extração do gipso; preparação para calcinação; calcinação e seleção. A gipsita é o mineral compacto de baixa dureza, pouco solúvel em água, que é a matéria prima para o gesso; sua fórmula química é CaSO4.2H2O.

A escolha do o gesso para o estudo se deve a vantagosa abundância, processo de fabricação e ao baixo custo, a capacidade do material agregar com maior ou menor valor a variados produtos utilizados nas atividades industriais e construção civil. No entanto, a pesquisa objetiva agrega-lo a fibra do babaçú, de forma sustentável, como isolamento acústico em painéis de gesso. Conforme Rezende, Filho e Nascimento (2014) o conceito de isolação sonora se refere à redução da transmissão sonora entre ambientes através de materiais ou sistemas construtivos que funcionem como barreiras físicas à propagação das ondas. Isolar acusticamente determinado recinto nada mais é que submetê-lo a um tratamento capaz de reduzir os ruídos externos a parâmetros compatíveis com a atividade nele desenvolvida (CARVALHO, 2010). Para o autor, a medição dos níveis de ruído, a unidade adotada é o decibel $(\mathrm{dB})$, onde um decibel equivale a um décimo de um bel.

Dentre as formas de isolamento acústico utilizando-se gesso, segundo Rezende, Filho e Nascimento (2014), há um método capaz de promover a isolação sonora entre áreas adjacentes é a utilização do sistema construtivo massa-mola-massa, representado na forma do drywall. Como o próprio nome induz, a constituição do sistema massa-mola-massa se dá pela junção de duas chapas - de gesso, por exemplo - e um espaço preenchido por ar ou outro material de elevada absorção sonora entre ambas (REZENDE, FILHO, NASCIMENTO, 2014). 
Com base nesse material, realizar os estudo sobre o isolamento sonoro faz-se necessário apresentar os requisitos, critérios e métodos de avaliação da ABNT NBR 15575 quanto ao desempenho acústico de sistemas de vedações verticais internas em edificações habitacionais sob o aproveitamento sustentável das fibras do babaçú para formação da placa de gesso.

A produção de placas de gesso sustentáveis se torna uma alternativa, com aproveitamento de resíduos de diferentes origens, como o babaçu, que pode contribuir para o atendimento da gestão de resíduos descartados nos espaços urbanos e industriais. A reutilização dos resíduos estimularia a produção de novos materiais de forma a atender as condições de sustentabilidade como alternativa econômica gerando placas ou painéis para uso arquitetônico ou na construção civil. O fato de que o uso de resíduos de babaçu venha a contribuir para minimizar os impactos ambientais.

Seguindo essa linha de proposta, esta pesquisa estuda a aplicação e produção de placas de gesso agregando a utilização das fibras da casca do babaçu como componente alternativo complementar e sustentável. A escolha do material para pesquisa é devido ao acesso de fabricação do gesso, facilidade de agregar a outros materiais segundo acompanhamento de ensaios laboratoriais, ainda que não exista uma norma específica para este estudo específico.

As placas de gesso e o drywal são componentes de uso interno, de divisória de ambientes e possui propriedades térmicas e acústicas. Embasado nisso, inicia-se um estudo dessas combinações de materiais, criando a placa de gesso com fibra de babaçu, buscando agregar com eficiência as exigências da construção civil e do meio ambiente contribuindo para um melhor conforto ambiental.

Com o estudo proposto, busca-se expandir as propriedades de isolamento acústico do gesso, combinando a fibra do babaçú como agregado, ocupando espaço e reduzindo o volume de gesso. Justifica-se dessa forma como o material ligante, tratando como uma malha a placa de gesso, diminuindo riscos de acidentes em eventuais rupturas.

Neste contexto, o artigo tem como objetivo avaliar a aplicação sustentável das fibras do babaçu a partir das propriedades de isolamento acústico com o gesso.

\section{Material e Método}

A sequência metodológica adotada na pesquisa foi desenvolvida em 04 (quatro) etapas. em seguida foi descrita a atividade realizada em cada uma das etapas:

1a etapa: Esta etapa consistiu em levantamento bibliográfico sobre composição e obtenção do gesso, origem e características de babaçu, placas de gesso e desempenho acústico a fim de subsidiar as informações tratadas nesse estudo.

2a etapa: Constitui a etapa de campo que envolveu a coleta dos 57 cocos de babaçu, frutos obtidos da Serra de Maranguape (Figuras 1).


Figura 1 - Mapa da localização de Maranguape (Ceará) Fonte: maranguapefotos.blogspot

Após coletados, os cocos de babaçu foram acondicionados em sacos plásticos e encaminhados para o processo de trituração da empresa a ENEL através de sua máquina de poda de árvores, cedidas para este fim, contribuindo na quebra dos cocos, como mostrado na Figura 2 abaixo. 

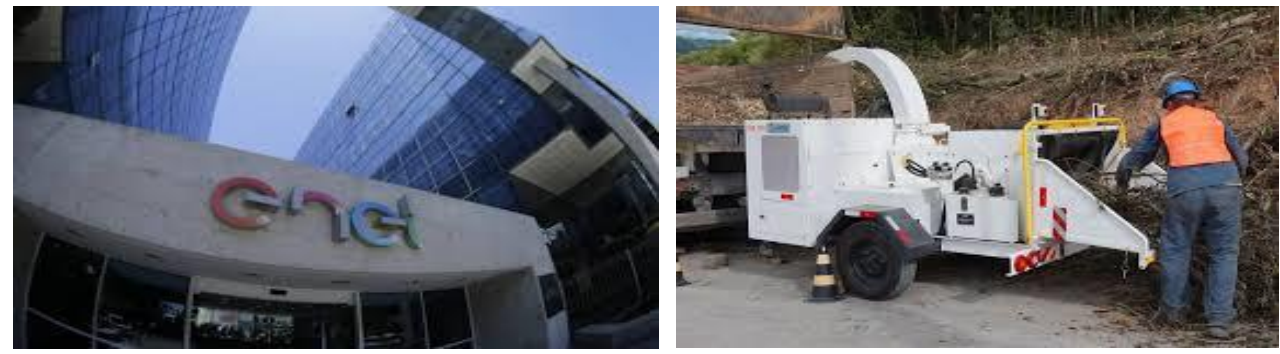

Figura 2 - A. ENEL (Ceará) (à esquerda); B. Máquinas de poda de árvores da ENEL (à direita) Fonte: Autora (2019)

3a etapa: A parte experimental constitui a terceira etapa da pesquisa, a qual foi subdividida em duas partes. A primeira parte trata-se da obtenção de placa contendo os elementos de gesso e das fibras do babaçu, componente agregado e objeto de estudo para o ensaio acústico do sistema de placa, ambos seguindo normas correspondentes. Vale ressaltar, que não há norma vigente que regulamente um ensaio apropriado como para materiais alternativos como babaçu. Dessa forma, o ensaio com o babaçu constituiu em adaptações de procedimentos laboratoriais da própria norma ABNT correspondente ao gesso e realizando-se assim os ensaios pertinentes para as fibras do coco do babaçu.

A segunda parte desta terceira etapa foi caracterizado pelos ensaios laboratoriais no Laboratório de Mecânica dos Solos e em sequência no Laboratório de Materiais da Construção Civil da mesma instituição. E conforme exposto, realizou-se adaptações da norma ABNT do gesso para as determinações da fibra do babaçu (Orbignya sp). Dessa forma, utilizou-se apenas a determinação das propriedades físicas do gesso na forma de pó, denominadas: a) granulometria; e um ensaio da temperatura na estufa, com dois tipos de amostras: fibra maior e fibra menor.

4a etapa: A organização dos procedimentos laboratoriais segundo a norma ABNT definiu a quarta etapa, contribuindo dessa forma, no desenvolvimento dos procedimentos seguintes resultando em valores a serem discutidos posteriormente. As etapas seguintes seguiram a seguinte sequência de ensaios: 1) Ensaio da Temperatura na Estufa (Figura 3); 2) Ensaio para determinação das propriedades físicas do gesso na forma de pó, denominadas de seguinte maneira: a) granulometria, b) massa unitária, segundo a norma especifica ABNT NBR 12127:1991; 3) Ensaio da produção da placa de gesso agregado ao babaçu, cujas dimensões da placa de madeira foi 40x40 (Figura 4); 4) Ensaio na determinação das propriedades mecânicas, que estabelece o método para determinação das propriedades mecânicas do gesso para construção, denominadas como: a) dureza, b) resistência à compressão, segundo a norma especifica ABNT NBR 12129:2017; 5) Ensaio acústico segundo a norma especifica ABNT NBR 15575 (Figura 5).
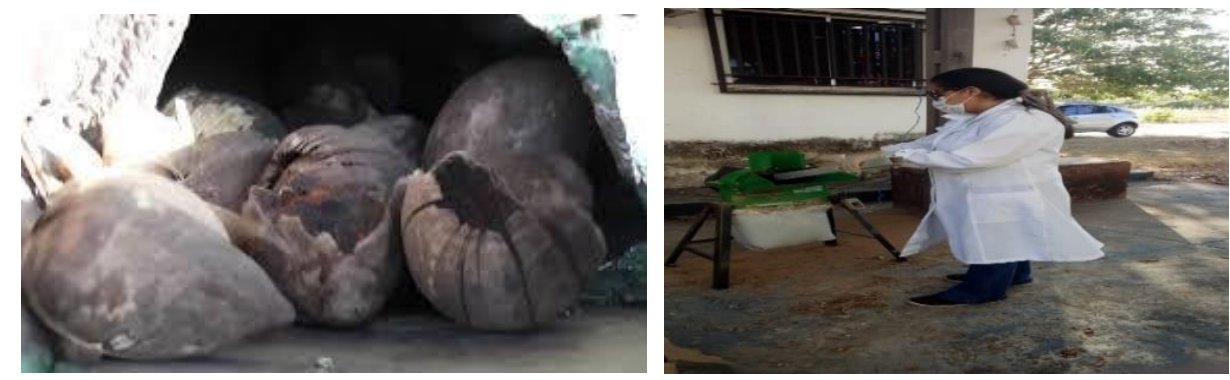

Figura 3 - A. Babaçu (Orbignya sp) (à esquerda); B. Máquinas de triturar do Laboratório de Física (na UECE) (à direita) Fonte: Autora (2019) 

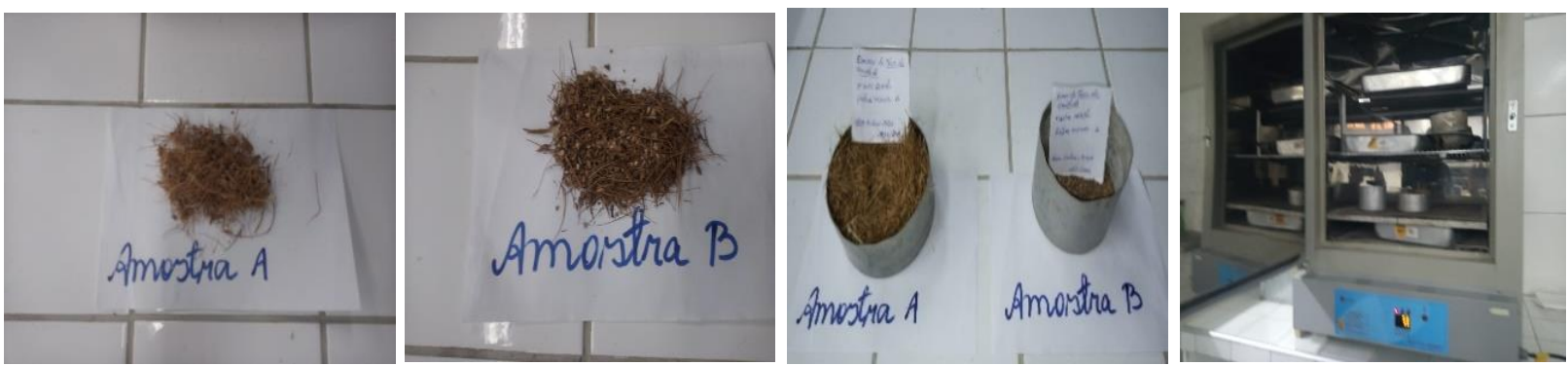

Figura 4 - A. Amostras da trituração do babaçu: fibra maior (A) e fibra menor (B) (à esquerda); Amostra nas cápsulas (no centro); B. Ensaio da Temperatura: amostras na Estufa (à direita).Fonte: Autora (2019)
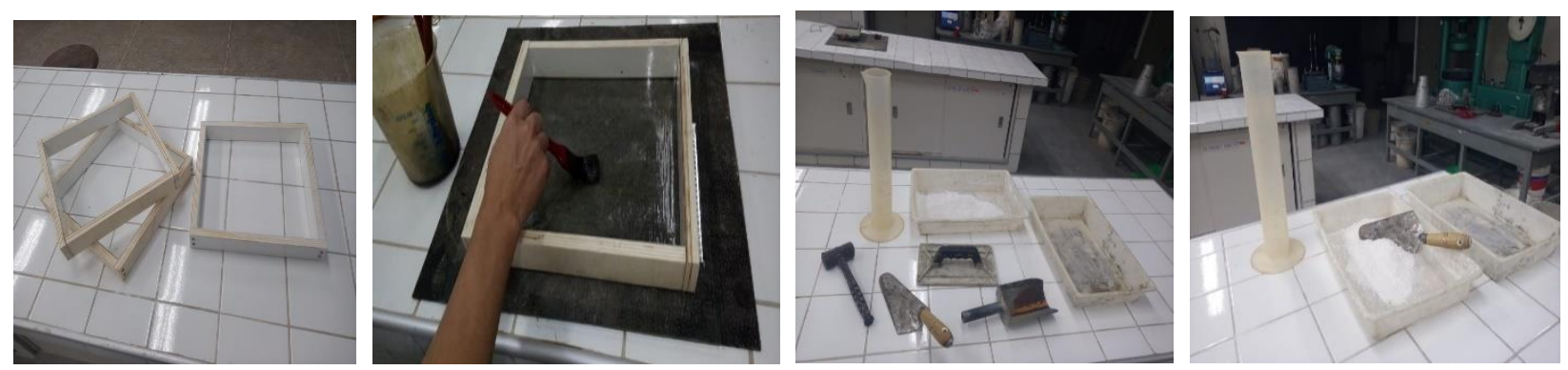

Figura 5 - Ensaio da produção da placa de gesso agregado ao babaçu. Fonte: Autora (2019)

Em seguida apresenta-se o fluxograma das etapas da sequência metodológica utilizada na pesquisa (Figura 6).

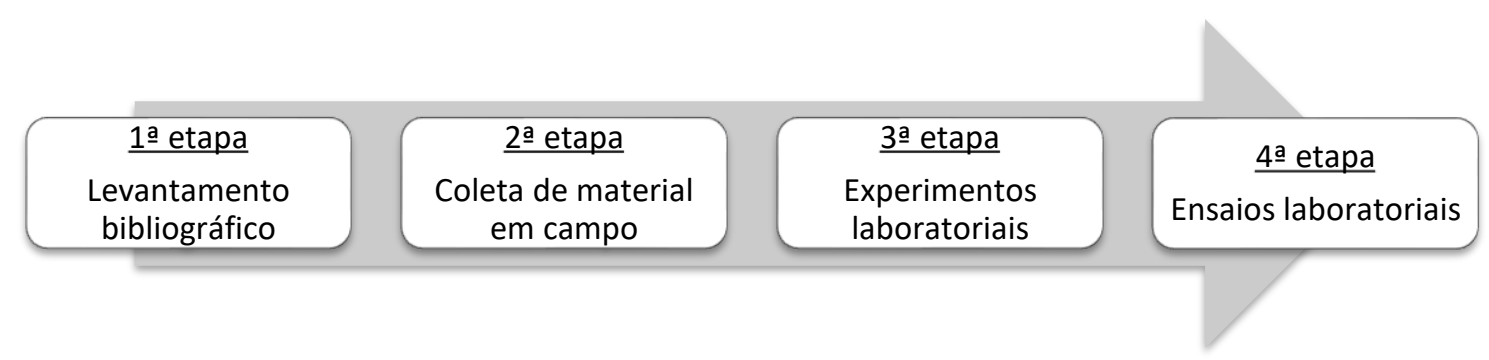

Figura 6 - Fluxograma das etapas metdológicas

\section{Resultados e Discussões}

Os resultados mostraram a partir da trituração do babaçu dois tipos de amostras em fibras, em função das hélices de trituração, gerou uma fibra maior (amostra A) e uma fibra menor (amostra B), visto na figura 6 abaixo. A partir de ambas foram pesados inicialmente amostra A com 107,9 e amostra B com 106,0 , ao ser submetido a estufa o peso final não variou tão significativamente amostra A com 107,2 e amostra B com 106,0, no entanto, pode contribuir para menor teor de umidade durante agregação do gesso. 

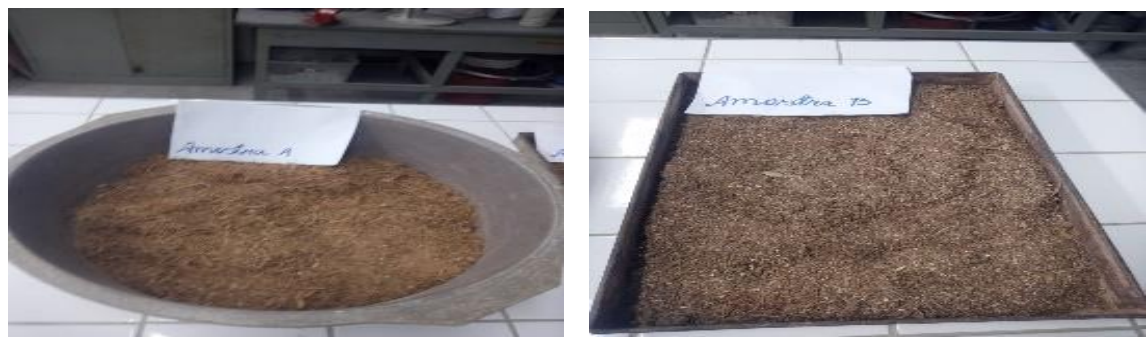

Figura 7 - Fibra maior (amostra A) e uma fibra menor (amostra B).Fonte: Autora (2019)
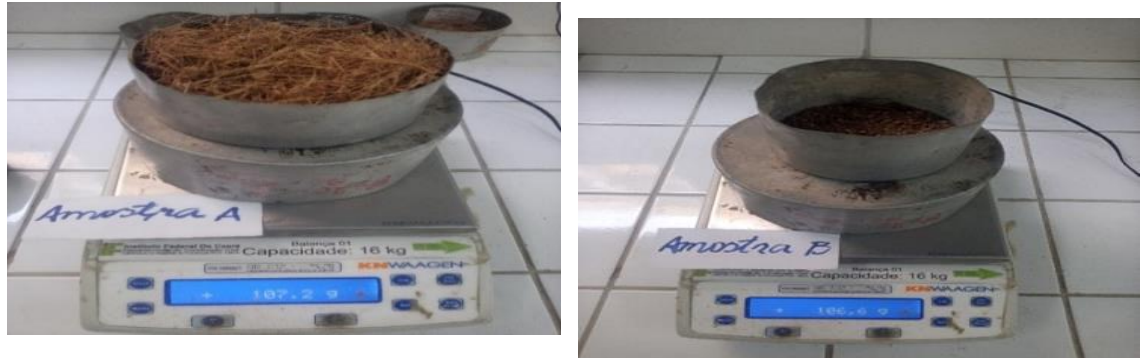

Figura 8 - Amostra A (á esquerda); amostra B (á direita) pós estufa Fonte: Autora (2019)

Nos ensaios de granulometria obteve como resultado o módulo de finura da amostra $A 5,47$, onde neste valor obtido se deve a partir da soma de massa retida acumulada referente as peneiras 6,3 até 0,15 além do fundo. Enquanto na amostra B 3,37 a partir da variação de peneiramento, visto na figura 8. Já na dimensão máxima, cujas características é em $\mathrm{mm}$, a amostra $A$ houve ausência deste valor devido não haver dado maior que $5 \%$ no material obtido.

No entanto, na amostra B 4,74 foi obtido a 0,75 , pois foi o primeiro valor maior que $5 \%$. Logo a peneira que representa será de $4,75 \%$. Destacando não haver norma especifica para materiais alternativos, sendo utilizado a norma de peneiramento agregado muido.

Os ensaios de acústica não foram viáveis no prazo devido ao tempo de cura do gesso com as fibras do babaçu a temperatura ambiente observado na figura 8 , e, portanto, não obtendo os dados esperados. Os ensaios foram submetidos a norma correspondente, porém de forma parcial, visto que a presente pesquisa está sob estudo e andamento.
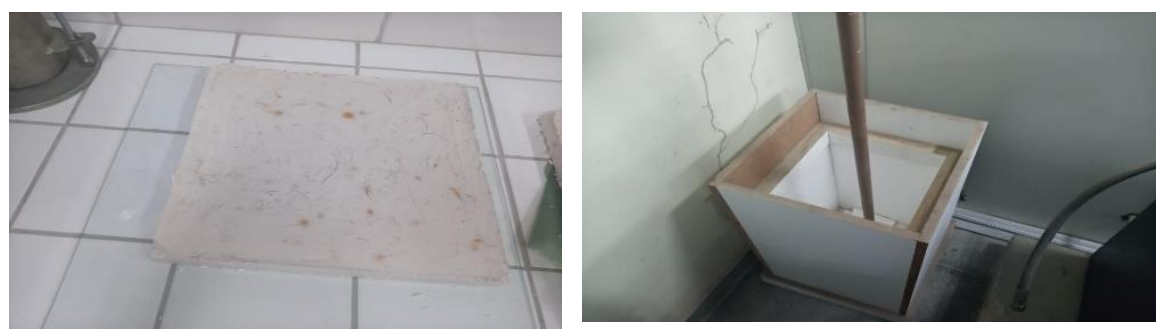

Figura 9 - A. Tempo de cura do gesso com as fibras do babaçu (à esquerda). B. Ensaios de acústica Fonte: Autora (2019)

\section{Conclusões}

Nos resultados foi possível notar nos ensaios da inserção da fibra que a amostra B pode contribuir na menor teor de umidade agregação do gesso. No entanto, ressalta-se a continuidade de ensaios específicos que venha atender a norma pertinente a ABNT NBR 12129:2017 e a norma ABNT NBR 12127:1991, bem como, critérios e métodos de avaliação da ABNT NBR 15575 que melhor adequa as expectativas de ensaio acústico com o aproveitamento sustentável de dos resíduos da fibra do babaçu. 
Dessa forma, mesmo antes da realização da análise do sistema construtivo sustentável em comparação com os parâmetros de adaptação no espectro sonoro, em teste acústico e sua respectiva adequação a norma, foi perceptível um impacto significativo para um possível desempenho acústico. Concluiu -se que nos ensaios pertinentes a serem realizados: propriedade mecânica, ensaio da determinação de dimensão e propriedades físicas e ensaio da determinação de aderência a tração ambos estão previstos a serem realizados posteriormente a fim de obter dados mais precisos para melhor avaliar qualidade da matéria-prima e do gesso.

No entanto, o estudo sugere continuidade a fim de melhor representar a viabilidade sustentável e na economia do reaproveitamento dos resíduos da fibra do coco do babaçu na forma de gesso e adequar as expectativas de ensaio acústico relevante a placa de gesso com o aproveitamento dos resíduos da fibra do babaçu.

\section{Agradecimentos}

Aos colegas e colaboradores do Laboratório de Física da Universidade Federal do Ceará -UECE.

Aos colegas e colaboradores do Laboratório de Mecânica dos Solos (LMC) do Instituto Federal do Ceará (IFCE).

Aos colegas e colaboradores do Laboratório de Materiais da Construção Civil (LMCC) do Instituto Federal do Ceará (IFCE).

\section{Referências Bibliográficas}

Associação Brasileira de Drywall. Desempenho acústico em sistemas drywall. 2013, 23p.

ABNT: Associação Brasileira de Normas Técnicas. NBR 15 575: Edificações habitacionais - Desempenho. Rio de Janeiro, 2013, $381 \mathrm{p}$.

ABNT: Associação Brasileira de Normas Técnicas. ABNT NBR 12129:2017: Edificações habitacionais Desempenho. Rio de Janeiro, 2013, 381 p.

ABNT: Associação Brasileira de Normas Técnicas. ABNT NBR 12127:1991: Gesso para construção Determinação das propriedades físicas do pó - Método de ensaio. Rio de Janeiro, 2013, 381 p.

Barbosa, A. A.; Ferraz, A. V. and Santos, G. A. (2014). Caracterização química, mecânica e morfológica do gesso $\beta$ obtido do pólo do Araripe. Cerâmica, vol.60, n.356, pp.501-508. ISSN 0366-6913.

Carvalho, R. P. Acústica arquitetônica. 2 ed. Brasília: Thesaurus Editora, 2010. 238 p.

Empresa Brasileira de Pesquisa Agropecuária. (1984). Babaçu: Programa Nacional de Pesquisa. Departamento de Orientação e Apoio à Programação de Pesquisa Brasileira. Brasília, DF. EMBRAPADDT, p. 39.

Instituto brasileiro de geografia e Estatística. (2016). Produção da Extração Vegetal e da Silvicultura 2016. Rio de Janeiro: IBGE, 2016. Disponível em: https://sidra.ibge. gov.br/pesquisa/pevs/quadros/brasil/2016. Acesso em: 15 fev. 2018.

Rezende, J. M. S.; FILHO, J. C. G. de M.; Nascimento, N. L. F. (2014). Isolamento Sonoro contra ruído aéreo de vedações verticais internas medido em campo. Trabalho de conclusão de curso. Escola de Engenharia Civil. Universidade Federal de Goiás, Goiânia.

Oliveira, A. I. T. de; Alexandre, G. P; Mahmoud, T. S. (2013). Babaçu (Orbignya sp): Caracterização física de frutos e utilização de solventes orgânicos para extração de óleo. Universidade Federal do Tocantins, Araguaína; v. 2, n. 3, p. 126-129. 